\title{
Ultrahigh-Order Wave Mixing in Noncollinear High Harmonic Generation
}

\author{
J. B. Bertrand, ${ }^{1}$ H. J. Wörner, ${ }^{1,3}$ H.-C. Bandulet, ${ }^{2}$ É. Bisson, ${ }^{2}$ M. Spanner, ${ }^{1}$ J.-C. Kieffer ${ }^{2}$ \\ D. M. Villeneuve, ${ }^{1}$ and P. B. Corkum ${ }^{1}$ \\ ${ }^{1}$ Joint Attosecond Science Laboratory, 100 Sussex Drive, Ottawa, K1A OR6, Canada \\ ${ }^{2}$ Institut National de la Recherche Scientique, 1650 boulevard Lionel-Boulet, Varennes, Québec J3X 1S2, Canada \\ ${ }^{3}$ Laboratorium für physikalische Chemie, ETH Zurich, Wolfgang-Pauli-Strasse 10, 8093 Zurich, Switzerland
}

(Received 28 September 2010; published 11 January 2011)

\begin{abstract}
We show that noncollinear high harmonic generation (HHG) can be fully understood in terms of nonlinear optical wave mixing. We demonstrate this by superposing on the fundamental $\omega_{1}$ field its second harmonic $\omega_{2}$ of variable intensity in a noncollinear geometry. It allows us to identify, by momentum conservation, each field's contribution $\left(n_{1}, n_{2}\right)$ to the extreme ultraviolet emission at frequency $\Omega=n_{1} \omega_{1}+n_{2} \omega_{2}$. We observe that the photon $(\Omega)$ yield follows an $n_{2}$ power law on the $\omega_{2}$ intensity, before saturation. It demonstrates that, although HHG is a highly nonperturbative process, a perturbation theory can still be developed around it.
\end{abstract}

DOI: 10.1103/PhysRevLett.106.023001

PACS numbers: $33.20 .-\mathrm{t}$

Perturbative nonlinear optics [1], introduced in the 1960s [2], arises from the interaction of light with materials where the response is described by perturbation theory. That is, only a few photons are involved and higher order processes are less important than lower ones, providing the lower one is allowed. Perturbative nonlinear optics is critical for short pulse science and technology. For example, the nonlinear response of materials is exploited in all pump-probe spectroscopy. Major technologies rely on nonlinear optics, optical communications being only one [3].

Nonperturbative nonlinear optics, introduced in the 1990s [4,5] for high harmonic generation (HHG), treats light interaction with materials nonperturbatively. Often the interaction is approximated with classical physicsphotons are not even needed to qualitatively understand the phenomena. Nonperturbative nonlinear optics has led to attosecond pulse formation [6] and new methods of imaging matter [7-9]. These two major trends-perturbative and nonperturbative nonlinear optics-seem conceptually very different.

Our experiment and analysis show that a perturbative nonlinearity can be fabricated on a highly nonperturbative nonlinear process. This opens a route to transfer the powerful techniques of perturbative nonlinear optics to high intensity, highly nonlinear phenomena such as a broad range of novel noncollinear HHG experiments.

To demonstrate this, we perform an experiment in which an intense fundamental laser pulse of frequency $\omega_{1}$ and a weak second harmonic field $\omega_{2}$ are noncollinearly crossed in a gas jet target. We show that the positions of discrete harmonics in the HHG spectrum can be described simply in terms of conservation of energy, momentum, and parity, based on the net number of photons $\left(n_{1}, n_{2}\right)$ absorbed from each field. That is, $\Omega=n_{1} \omega_{1}+n_{2} \omega_{2}$ and $\vec{k}_{\Omega}\left(n_{1}, n_{2}\right)=$ $n_{1} \vec{k}_{1}+n_{2} \vec{k}_{2}$. By studying the intensity dependence of the harmonic yield for a particular combination $\left(n_{1}, n_{2}\right)$, we find that, at low intensity $I_{2}$ of the weak field, the harmonic signal scales as $I_{2}^{n_{2}}$ and that each higher order in $n_{2}$ is less important than the lower one-just as we expect from perturbation theory. Therefore, HHG is nonperturbative regarding the high intensity fundamental beam and it is perturbative for the much weaker second harmonic beam.

Our work builds on a long history of experiments on using multiple color fields to produce high harmonics [10-18]. Early experiments confirmed that energy, momentum, and parity are preserved when high harmonics are generated in two-color fields [10]. This introduced the potential to control the high-harmonic process [11-13], leading to the generation of attosecond pulse trains with individual pulses separated by the laser period [14] and new approaches to isolating a single attosecond pulse [15-17] and generating tunable extreme ultraviolet (XUV) radiation [18]. Recently, it has been shown that weak-field control of high harmonic generation allows attosecond pulses to be measured as they are being produced in the medium [19]. Tacitly, in situ measurement suggests an ability to construct a perturbative nonlinear optics theory upon the physics of high harmonic generation.

Noncollinear techniques were also used previously mainly to separate high-harmonic radiation from the fundamental beam [20-22]. An important recent application is in extreme ultraviolet frequency comb metrology where a noncollinear control pulse switches high-harmonic radiation from a buildup cavity [23].

The experimental setup is composed of a Ti:sapphire multipass laser system $(35 \mathrm{fs}, 800 \mathrm{~nm}, 50 \mathrm{~Hz}, 10 \mathrm{~mJ}$ per pulse), an optical setup to both split and recombine laser pulses, and a high-harmonic chamber composed of a source chamber (pulsed valve, $250 \mu \mathrm{m}$ orifice), an XUV grating, a microchannel plate detector, and a camera readout. The laser output is divided using a 50/50 beam splitter. 
In one arm of the Mach-Zehnder interferometer, second harmonic of the fundamental laser field is generated in a type-I BBO crystal of $100 \mu \mathrm{m}$ thickness and the remaining fundamental light is removed by two reflections on $400 \mathrm{~nm}$-only reflective mirrors. In both arms, the intensity is adjusted with neutral absorption-type density filters. The fundamental $\omega_{1}$ and its second harmonic $\omega_{2}$ are then recombined using a dichroic mirror, where $\omega_{2}$ is reflected and the beams are spaced vertically by $7 \mathrm{~mm}$. Finally, both beams are focused $(f=50 \mathrm{~cm}) \sim 2 \mathrm{~mm}$ before and $\sim 1 \mathrm{~mm}$ downstream of the gas jet $\left(N_{2}\right)$ in the source chamber [Fig. 1(a)].

Figure 1(b) shows the XUV emission resulting from the superposition of a weak second harmonic field $\omega_{2}(400 \mathrm{~nm}$, $I_{2}=1.5 \times 10^{13} \mathrm{~W} / \mathrm{cm}^{2}$ ) crossing a strong fundamental field $\omega_{1}\left(800 \mathrm{~nm}, I_{1}=1.5 \times 10^{14} \mathrm{~W} / \mathrm{cm}^{2}\right)$ at an angle $\phi \approx 14 \mathrm{mrad}$ [see schematic in Fig. 1(a)]. The fundamental alone generates a high-harmonic spectrum along the $\theta=0$ axis shown by the dashed line. The addition of the weak field gives rise to off-axis radiation at various discrete angles in the vertical direction. We use conservation of energy and momentum to determine the contribution of each field $\omega_{1,2}$ to the resulting XUV emission. For example, the photon centered at $\Omega=13 \omega_{1}$ in the direction $\theta=8.4 \mathrm{mrad}$ has a unique solution given by

(a)
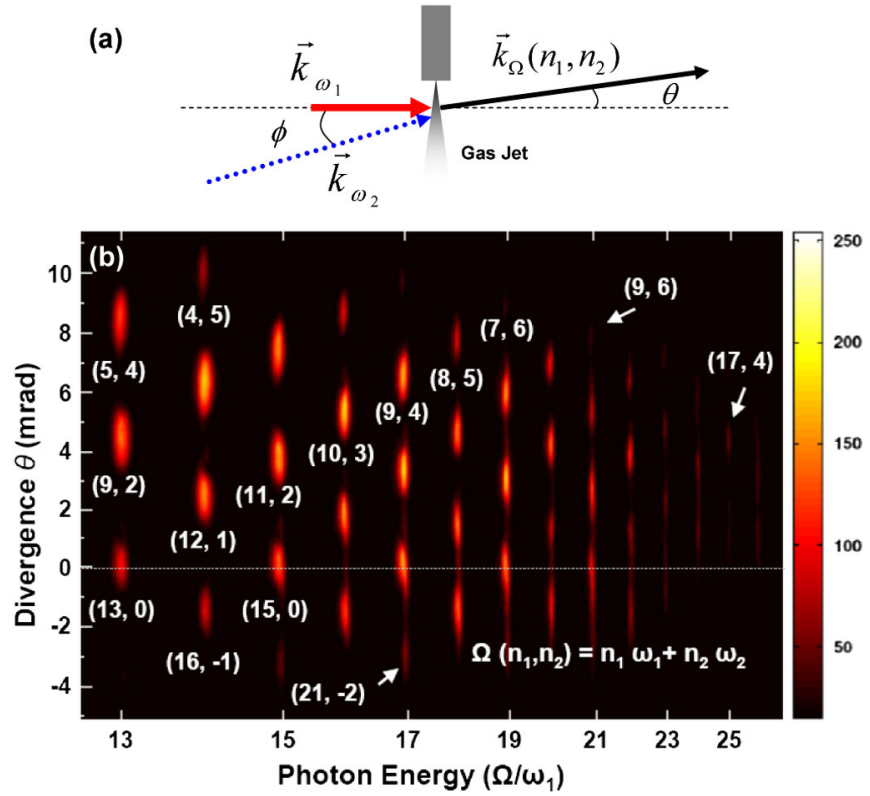

FIG. 1 (color online). Noncollinear harmonic generation from the superposition of an intense fundamental $\omega_{1}(800 \mathrm{~nm}$, $\left.I_{1}=1.5 \times 10^{14} \mathrm{~W} / \mathrm{cm}^{2}\right)$ and weaker $\omega_{2}\left(400 \mathrm{~nm}, I_{2}=\right.$ $1.5 \times 10^{13} \mathrm{~W} / \mathrm{cm}^{2}$ ) fields in randomly aligned $\mathrm{N}_{2}$ gas. (a) Schematic representation: The $\omega_{1}$ beam is in the horizontal plane and the $\omega_{2}$ beam points upwards at an angle $\phi \approx 14 \mathrm{mrad}$ such that they form a plane parallel to the entrance slit of the XUV spectrometer. (b) XUV emission as seen on the spectrometer detector. Each field's contribution is labeled as $\left(n_{1}, n_{2}\right)$ at frequency $\Omega=n_{1} \omega_{1}+n_{2} \omega_{2}$. $\left(n_{1}, n_{2}\right)=(5,4)$. As the intensity of the weak field increases, the off-axis emission increases away from $\theta=0^{\circ}$, but the positions of the harmonic peaks $\left(\Omega / \omega_{1}, \theta\right)$ do not change.

High-harmonic radiation comes from the recombination of an electron back to its ground state [4]. Since a single XUV photon is emitted through this dipolar transition, parity conservation requires that the wave function of the continuum electron and the ion together be in a state of different parity $(+$ or -$)$ than the original ground state $(-$ or +$)[10,24]$. Consequently, only the net absorption of an odd total number of photons $n=n_{1}+n_{2}$ can lead to emission. From this approach, it is possible to produce the same frequency but with various $\left(n_{1}, n_{2}\right)$ combinations. For example, see Fig. 2(a), $\Omega=8 \omega_{1}$ can be obtained from the net sum $\left(n_{1}, n_{2}\right)=(6,1)$ but also the net difference $(10,-1)$ frequency-mixing pathways. The noncollinear scheme offers the advantage of distinguishing them spatially as required by momentum conservation; see Fig. 2(b). We now understand the alternate appearance of even and odd harmonics as a function of $\theta$ in Fig. 1: an $n_{2}$ odd (even) number of photons involved from the weak field results in the observation of even (odd) harmonic orders.

Next, keeping the intensity of the strong $\omega_{1}$ beam fixed $\left(1.5 \times 10^{14} \mathrm{~W} / \mathrm{cm}^{2}\right)$, we measure spectra for various peak intensities of the weak $\omega_{2}\left[(0.1-7) \times 10^{13} \mathrm{~W} / \mathrm{cm}^{2}\right]$. In Fig. 3(a), we plot, on a log-log scale, the spatially $(\theta)$ and spectrally $\left(\Omega / \omega_{1}\right)$ integrated signal for different values of $n_{2}$ from 0 to 6 . This scaling allows us to determine the order of nonlinearity connecting the noncollinear XUV yield with the weak-field intensity.

(a)

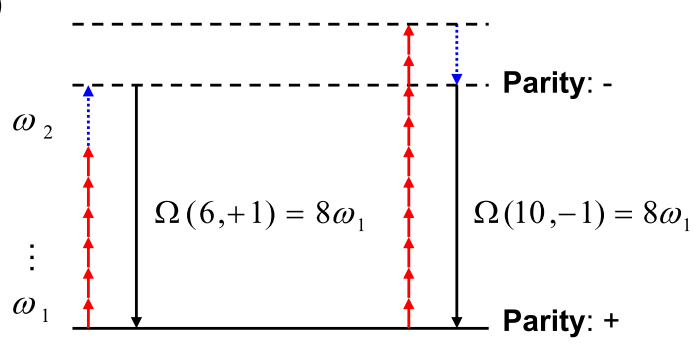

(b)

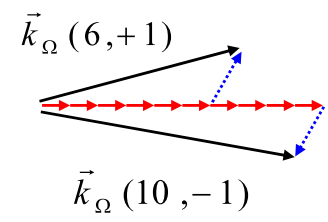

FIG. 2 (color online). Frequency-domain perspective on twocolor noncollinear HHG. (a) Sum and difference frequency emission $\Omega=n_{1} \omega_{1}+n_{2} \omega_{2}$ : only the odd net totals $n=$ $n_{1}+n_{2}$ are dipole-allowed transitions; see text. Illustrated is the production of $\Omega=8 \omega_{1}$ with $n_{2}= \pm 1$. (b) Momentum conservation yields phase-matched XUV radiation in the direction of $\vec{k}_{\Omega}\left(n_{1}, n_{2}\right)=n_{1} \vec{k}_{1}+n_{2} \vec{k}_{2}$. 

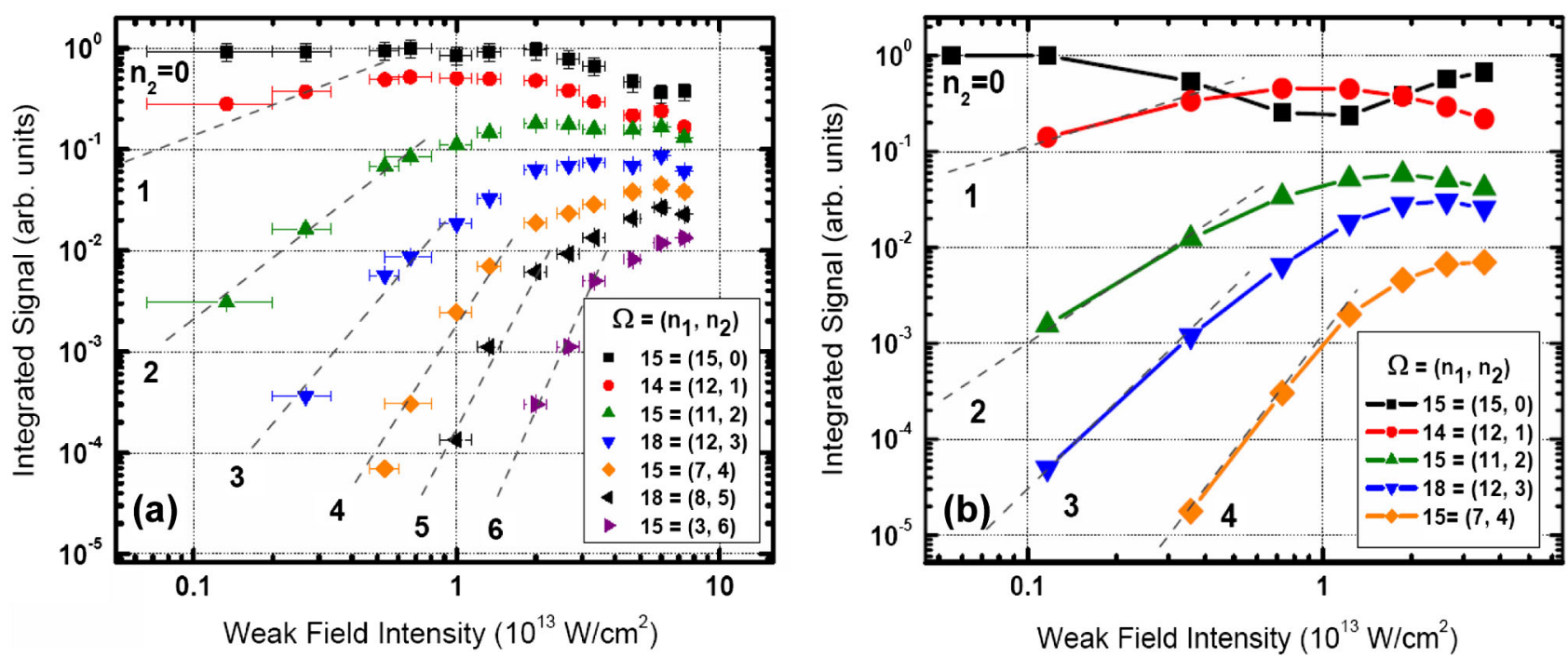

FIG. 3 (color online). (a) Experiment: Power scaling of the noncollinear harmonic emission as a function of the weak-field (400 nm) peak intensity $\left(I_{2}\right)$ for different numbers ( $n_{2}$ from 0 to 6 ) of weak $\omega_{2}$ field photons absorbed. The intensity of the fundamental $\omega_{1}$ field is kept constant $\left(I_{1}=1.5 \times 10^{14} \mathrm{~W} / \mathrm{cm}^{2}\right)$. The measured curves are labeled by $n_{2}$ and, respectively, multiplied vertically by a factor $(1,1 / 2,1 / 8,1 / 10,1 / 40,1 / 60,1 / 150)$ for clarity. Slopes of $n_{2}$ from 0 to 6 are also displayed (dashed line). (b) Theory: Calculated power scaling. Noncollinear XUV spectra are obtained by solving for two-color HHG in the laser focus on a 2D grid (plane formed by $\vec{k}_{1}+\vec{k}_{2}$, see text) and adding coherently the radiation in the far field. Each curve is scaled vertically by the same factors as in Fig. 3(a) and theoretical slopes of $n_{2}$ from 0 to 4 are displayed (dashed line).

We observe that for each value of $n_{2}$, the yield increases then saturates, the processes with lower $n_{2}$ saturating earlier. The values of $\Omega$ are chosen arbitrarily [see legend of Fig. 3(a)], emphasizing that the main shape of the curves for any $\left(n_{1}, n_{2}\right)$ is mainly dictated by $n_{2}$. We have also performed the same scaling with the $\omega_{2}$ field polarized perpendicular (not shown) to $\omega_{1}$ and observed a similar scaling. The main difference being that the off-axis emission is less ( $\approx 1 / 10$ for $\Omega(12,1)=14$ ) at the same intensity of $\omega_{2}$.

We deduce from the linear part of each curve that the photon yield follows a perturbative power law of the form $I_{2}^{n_{2}}$. This behavior is seen in optical wave mixing in media in which a time-dependent nonlinear polarization is induced [1]. The formalism behind it, perturbation theory, is not intuitively applicable to a highly nonperturbative process such as HHG. Cascaded wave mixing has been proposed [25] to explain the new frequency components, where the initial odd harmonics undergo four-wave mixing with the remaining $\omega_{1,2}$ fields. Next, we find instead that the perturbative power-law scaling of the form $I_{2}^{n_{2}}$ finds its origin directly in the single-atom HHG process.

We perform a two-dimensional calculation incorporating both the single-emitter response and the macroscopic noncollinear overlap of $\omega_{1,2}$ beams as in the experiment. At each point on a $2 \mathrm{D}$ grid (plane formed by $\vec{k}_{1}+\vec{k}_{2}$ ), we solve for the time-dependent XUV fields produced by the two noncollinear beams. We use the strong field approximation formalism as described in [26]. The grid size was 400 (diameter) by 800 (axial) points, creating a source size of $100 \times 200 \mu \mathrm{m}$. We coherently sum the emission from each grid point on the detector in the far field at a distance $L=80 \mathrm{~cm}$ and generate the power spectrum. The calculated noncollinear HHG spectra are very similar to what is measured experimentally [see Fig. 1(b)]; i.e., the positions of the various harmonic orders were correctly predicted in both frequency and angle.

In Fig. 3(b), we vary the intensity $I_{2}$ of the weak field and evaluate the integrated XUV emission of various $\Omega\left(n_{1}, n_{2}\right)$ points as in the experiment. In the low intensity range, we reproduce the perturbative $I_{2}^{n_{2}}$ nonlinear dependence observed experimentally [see Fig. 3(a)]. At higher intensity we also observe a saturation effect. Our simple model for noncollinear HHG predicts the perturbative $I_{2}^{n_{2}}$ scaling of the noncollinear $\Omega\left(n_{1}, n_{2}\right)$ emission. It shows that the yield of noncollinear harmonic emission due to an additional weak $\left(\omega_{2}\right)$ field can be understood essentially from the strong field approximation single-emitter perspective with macroscopic phase matching, and amounts to building a perturbation theory on HHG initially driven by a strong $\left(\omega_{1}\right)$ field alone.

A feature common to both theory and experiment is that the emission is always weaker for $n_{2}<0$ than for $n_{2}>0$, e.g., by more than 1 order of magnitude between the +1 and -1 orders in this experiment [see Fig. 1(b)]. Our calculated noncollinear XUV pattern also shows this updown asymmetry, but not as strongly. We observe that it depends weakly on the length of the medium used in the calculation. Further modifications, including the implementation of propagation effects [13], are needed to further 
investigate this aspect. Asymmetry between sum and difference frequency-mixing processes in HHG have been reported before in collinear experiments using incommensurate two-color $\omega_{1,2}$ fields $[17,27,28]$. They could originate from an interplay between the single-emitter response and propagation effects $[13,29]$.

In conclusion, we have confirmed that the framework of perturbative optical wave mixing applies to describe HHG modified by a weak laser field. First, we demonstrated that the laws of momentum, energy, and parity can predict the direction of the allowed emission. This view will be important in future experiments as four-wave-mixing-like noncollinear schemes, such as transient grating spectroscopy $[9,30,31]$, are migrating to attosecond science. Furthermore, noncollinear two-color HHG can act as an all-optical XUV beam splitter which could be implemented for XUV (pump)-XUV (probe) interferometry experiments. Second, our observation revealed an important property of the HHG process, which is that, although it is a highly nonperturbative process, a perturbative formalism can still be developed around it, up to ultrahigh orders of nonlinearity.

Our experiment bridges the conceptual gap between perturbative nonlinear optics and HHG. It opens new avenues for exploiting the powerful concepts of wave mixing: these include background-free detection of molecular dynamics and the probing of electronic coherence by HHG [9].

The authors thank A. Naumov, A. G. Avery, D. A. Crane, and R. M. Kroeker for their support and assistance during the experiment. Funding from Canadian Institute for Photonic Innovation, NSERC, and AFOSR is also acknowledged.

[1] R. W. Boyd, Nonlinear Optics (Academic, New York, 2008), 3rd ed.

[2] N. Bloembergen and P. S. Pershan, Phys. Rev. 128, 606 (1962).

[3] T. Schneider, Nonlinear Optics in Telecommunications (Springer-Verlag, Berlin, 2004).

[4] P. B. Corkum, Phys. Rev. Lett. 71, 1994 (1993).

[5] M. Lewenstein, P. Balcou, M. Y. Ivanov, A. L'Huillier, and P. B. Corkum, Phys. Rev. A 49, 2117 (1994).

[6] E. Goulielmakis et al., Science 320, 1614 (2008).

[7] J. Itatani, J. Levesque, D. Zeidler, H. Niikura, H. Pépin, J. C. Kieffer, P. B. Corkum, and D. M. Villeneuve, Nature (London) 432, 867 (2004).

[8] O. Smirnova, Y. Mairesse, S. Patchkovskii, N. Dudovich, D. M. Villeneuve, P. B. Corkum, and M. Y. Ivanov, Nature (London) 460, 972 (2009).
[9] H. J. Wörner, J. B. Bertrand, D. V. Kartashov, P. B. Corkum, and D. M. Villeneuve, Nature (London) 466, 604 (2010).

[10] M.D. Perry and J. K. Crane, Phys. Rev. A 48, R4051 (1993).

[11] S. Watanabe, K. Kondo, Y. Nabekawa, A. Sagisaka, and Y. Kobayashi, Phys. Rev. Lett. 73, 2692 (1994).

[12] M. Y. Ivanov, P. B. Corkum, T. Zuo, and A. D. Bandrauk, Phys. Rev. Lett. 74, 2933 (1995).

[13] M. B. Gaarde, A. L'Huillier, and M. Lewenstein, Phys. Rev. A 54, 4236 (1996).

[14] J. Mauritsson, P. Johnsson, E. Gustafsson, A. L'Huillier, K. J. Schafer, and M.B. Gaarde, Phys. Rev. Lett. 97, 013001 (2006).

[15] T. Pfeifer, L. Gallmann, M. J. Abel, P. M. Nagel, D. M. Neumark, and S. R. Leone, Phys. Rev. Lett. 97, 163901 (2006).

[16] H. Mashiko, S. Gilbertson, C. Li, S. D. Khan, M. M. Shakya, E. Moon, and Z. Chang, Phys. Rev. Lett. 100, 103906 (2008).

[17] H.-C. Bandulet, D. Comtois, E. Bisson, A. Fleischer, H. Pépin, J.-C. Kieffer, P. B. Corkum, and D. M. Villeneuve, Phys. Rev. A 81, 013803 (2010).

[18] H. Eichmann, S. Meyer, K. Riepl, C. Momma, and B. Wellegehausen, Phys. Rev. A 50, R2834 (1994).

[19] N. Dudovich, O. Smirnova, J. Levesque, M. Y. Ivanov, D. M. Villeneuve, and P. B. Corkum, Nature Phys. 2, 781 (2006).

[20] J. Peatross, J.L. Chaloupka, and D. D. Meyerhofer, Opt. Lett. 19, 942 (1994).

[21] P. M. Paul, E. S. Toma, P. Breger, G. Mullot, F. Augé, P. Balcou, H. G. Muller, and P. Agostini, Science 292, 1689 (2001).

[22] S. V. Fomichev, P. Breger, B. Carré, P. Agostini, and D. Zaretsky, Laser Phys. 12, 383 (2002).

[23] A. Ozawa, A. Vernaleken, W. Schneider, I. Gotlibovych, T. Udem, and T. Hänsch, Opt. Express 16, 6233 (2008).

[24] A. Fleischer and N. Moiseyev, Phys. Rev. A 74, 053806 (2006).

[25] L. Misoguti, I. P. Christov, S. Backus, M. M. Murnane, and H. C. Kapteyn, Phys. Rev. A 72, 063803 (2005).

[26] V. S. Yakovlev, M. Ivanov, and F. Krausz, Opt. Express 15, 15351 (2007).

[27] M. B. Gaarde, P. Antoine, A. Persson, B. Carré, A. L'Huillier, and C.-G. Wahlström, J. Phys. B 29, L163 (1996).

[28] Y. Oguchi, S. Minemoto, and H. Sakai, Phys. Rev. A 80, 021804 (2009).

[29] P. Balcou, A.S. Dederichs, M. B. Gaarde, and A. L'Huillier, J. Phys. B 32, 2973 (1999).

[30] Y. Mairesse, D. Zeidler, N. Dudovich, M. Spanner, J. Lévesque, D. M. Villeneuve, and P. B. Corkum, Phys. Rev. Lett. 100, 143903 (2008).

[31] J. P. Farrell, L. S. Spector, M. B. Gaarde, B. K. McFarland, P. H. Bucksbaum, and M. Gühr, Opt. Lett. 35, 2028 (2010). 